\title{
An assessment of risk posed by a Campylobacter-positive puppy living in an Australian residential aged-care facility
}

\author{
Cameron Moffatt, ${ }^{a}$ Ranil Appuhamy, ${ }^{b}$ Will Andrew, ${ }^{c}$ Sandy Wynn, ${ }^{d}$ Jan Robertse and Karina Kennedy \\ Correspondence to Cameron Moffatt (e-mail: cameron.moffatt@act.gov.au).
}

Introduction: In April and June 2012, two outbreaks of Campylobacter gastroenteritis were investigated in an Australian aged-care facility (ACF); a Campylobacter-positive puppy was identified as a potential source of infection.

Methods: An expert panel was convened to assess transmission risk from the puppy to elderly residents and to guide further public health action. Criteria considered as part of the panel's assessment included the puppy's infectivity, the bacterium's transmissibility, puppy-resident contact, infection control and cleaning practices and animal management at the facility. A literature review was used to assist the panel, with a final risk being determined using a likelihood and consequence matrix.

Results: The panel determined that the setting and low infective dose made transmission likely despite varying degrees of contact between the puppy and cases. While infection control practices were generally appropriate, the facility's animal policy did not adequately address potential zoonotic risk.

Conclusion: In summary, puppies should not be considered as companion animals in ACFs due to high rates of Campylobacter carriage and the underlying susceptibility of the elderly. Infection control and animal policies in ACFs should reflect an awareness of zoonotic disease potential.

C ampylobacter is the most commonly notified cause of gastroenteritis in Australia, but few outbreaks are identified relative to disease incidence. ${ }^{1}$ As in other industrialized countries, the majority of Australian cases are attributed to foodborne transmission, with chicken consumption and raw poultry contact identified as significant risk factors for disease. ${ }^{2,3}$ Human illness generally manifests as an acute self-limiting enteritis with symptoms of diarrhoea, fever and abdominal pain; extra-intestinal manifestations, notably bacteraemia and sequelae such as Guillain-Barré syndrome and reactive arthritis may also occur. ${ }^{4}$

\section{Event Background}

Between 24 April and 25 June 2012, two outbreaks of Campylobacter gastroenteritis occurred at an
Australian residential aged-care facility (ACF) (Figure 1). These outbreaks affected 13 residents and two staff including five residents and one staff member with laboratory-confirmed campylobacteriosis. Inspections by environmental health officers and infection control staff identified no issues with either food safety or infection control practices. Following the second outbreak, investigators learnt of a four-month-old puppy living in the facility; the dog's arrival predated the initial outbreak by one week.

Investigation of the puppy revealed it had access to communal areas, residents' rooms and the dining room. Anecdotally staff reported close contact between the puppy and residents and staff. The animal's health was reported as good with no history of diarrhoeal illness. It was encouraged to toilet outside but on occasion

\footnotetext{
OzFoodNet, Communicable Disease Control Section, Health Protection Service, ACT Government Health Directorate, Canberra, Australia.

Office of the Chief Health Officer, ACT Government Health Directorate, Canberra, Australia.

Territory and Municipal Services Directorate, Canberra, Australia.

Infection Control, Health Protection Service, ACT Government Health Directorate, Canberra, Australia.

Infection Control, Community-based Services, ACT Health Directorate, Canberra, Australia.

Department of Microbiology and Infectious Diseases, Canberra Hospital and Health Services, Canberra, Australia.

Submitted: 12 May 2014; Published: 11 August 2014

doi: 10.5365/wpsar.2014.5.2.009
} 
Figure 1. Suspected animal-to-human outbreaks of Campylobacter gastroenteritis in an Australian aged-care facility, 2012

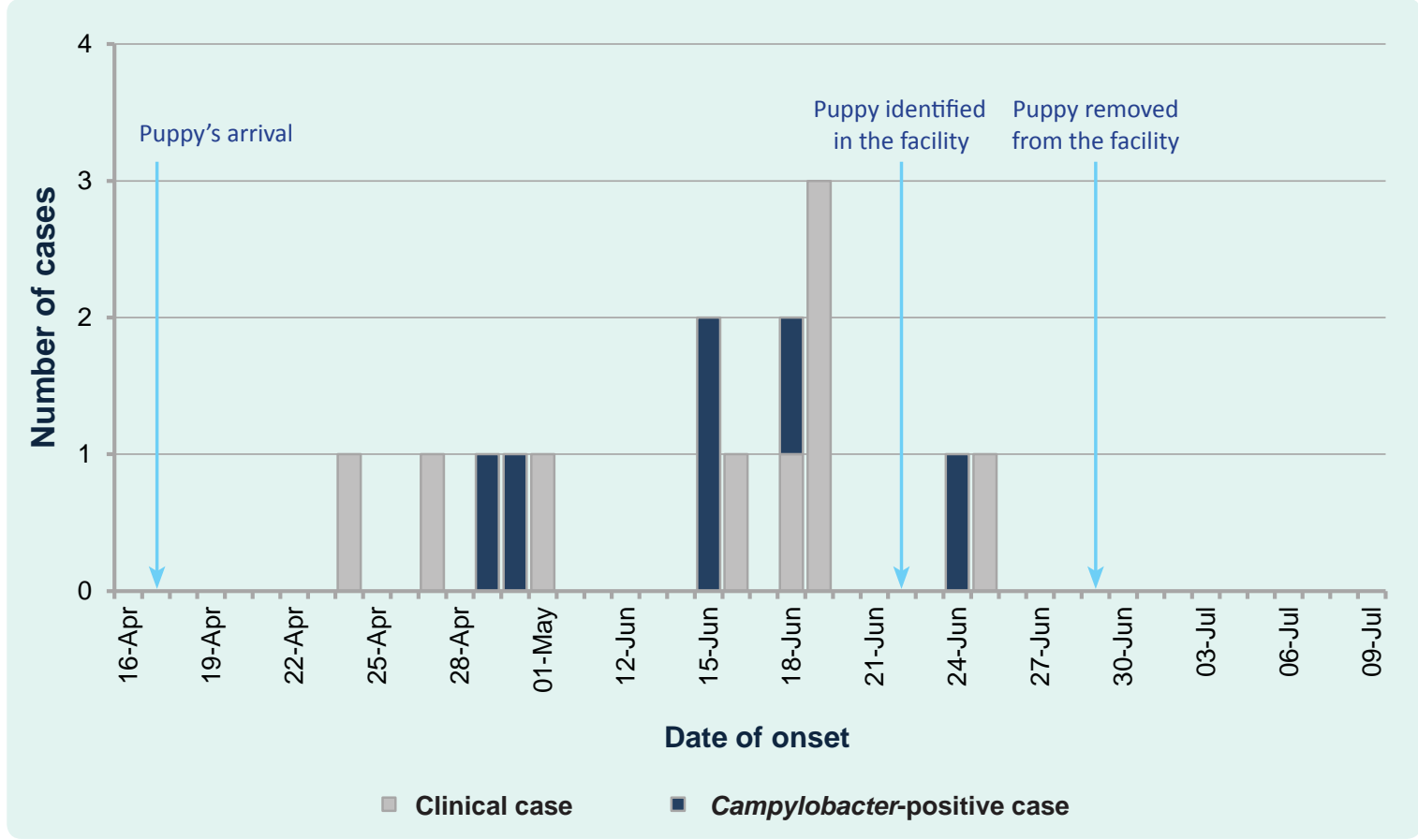

was reported to have defecated within the facility. Its diet consisted of commercial puppy biscuits and chews.

Pending further testing and assessment, public health advice was given to remove the puppy from the facility. In addition to laboratory-confirmed infections in people, a Campylobacter-positive faecal sample was recovered from the puppy. Both the human- and caninederived campylobacters were tested for relatedness via speciation, antibiotic susceptibility testing and pulsedfield gel electrophoresis (PFGE). Only one human isolate could be re-grown by the reference laboratory and was confirmed as a Campylobacter jejuni subspecies jejuni, with sensitivity to ciprofloxacin, nalidixic acid, gentamicin and tetracycline. The canine isolate was also identified as Campylobacter jejuni subspecies jejuni with resistance to ciprofloxacin and nalidixic acid. PFGE results showed an obvious heterogeneity between the human and canine isolates. While these results could not demonstrate a causal link between the puppy and human cases, the recovery of a significant human pathogen from an animal residing among a vulnerable population raised concerns of risk to residents' health.

\section{METHODS}

An expert panel was organized to assess the risk of Campylobacter transmission from the puppy to residents (and staff) at the ACF and to determine if additional public health actions should be taken. The panel comprised disciplines including epidemiology, public health medicine, veterinary medicine, infection control and medical microbiology. Panel members were asked to consider both risk assessment and risk management, specifically if the puppy posed a risk to residents' health and also if the puppy should be allowed back into the facility (and under what conditions).

To assess the risk of transmission from the puppy to residents, the panel members were asked to consider the puppy's infectivity, the bacterium's transmissibility, the level of contact between the puppy and cases, the current infection control and cleaning processes and current management of animals within the facility. A literature review was performed to provide a background hazard assessment, an examination of Campylobacter transmissibility and the consequences of the infection. To assist with ongoing risk management, the panel 
Figure 2. Risk assessment matrix*

\begin{tabular}{|c|c|c|c|c|c|c|}
\hline & \multirow{2}{*}{ LIKELIHOOD } & \multicolumn{5}{|c|}{ CONSEQUENCE } \\
\hline & & Insignificant & Minor & Moderate & Major & Catastrophic \\
\hline \multirow[t]{5}{*}{$\uparrow$} & Almost certain & Medium & High & High & Extreme & Extreme \\
\hline & Likely & Medium & Medium & High & High & Extreme \\
\hline & Possible & Low & Medium & Medium & High & Extreme \\
\hline & Unlikely & Low & Medium & Medium & High & High \\
\hline & Rare & Low & Low & Medium & Medium & High \\
\hline
\end{tabular}

* Adopted from Australian Capital Territory Health Directorate Risk Management Guidelines.

members were also asked to consider the potential impact of antibiotic therapy for the puppy as well as to review the facility's infection control and animal policies. The panel used a likelihood and consequence matrix to derive a final assessment of risk (Figure 2).

\section{RESULTS}

\section{Risk assessment}

\section{Likelihood of transmission of the bacterium from the puppy to residents and staff}

With animal-assisted interventions (AAI) in health settings now common, the potential for zoonotic transmission should be considered. ${ }^{5}$ Potential risk can be minimized by having clean, healthy, vaccinated, wellbehaved and trained animals. Thus service animals, like guide dogs, could be regarded as posing little threat to human health in ACFs with no published reports of infectious diseases that affect humans originating in this category of animal. ${ }^{5,6}$ However, the situation should be viewed differently with respect to puppies and young dogs in an ACF.

The panel assessed the following criteria to assist with determining the likelihood of Campylobacter transmission from the puppy:

\section{(1) Infectivity of the puppy}

Contact with puppies and young chickens have been identified as risk factors for campylobacteriosis in Australia, ${ }^{2,7}$ with an estimated 8500 cases being attributed to these two exposures annually. ${ }^{3}$ The biological plausibility of this is supported by evidence of shedding of Campylobacter spp. in both symptomatic and asymptomatic dogs with particular evidence of a correlation between younger dogs and the shedding of Campylobacter jejuni, ${ }^{8,9}$ the major species that affects humans.

The deficiencies in understanding what constitutes normal and abnormal canine intestinal microflora means that the patho-physiology of Campylobacter enteritis in dogs is not well understood. ${ }^{10}$ However, some Campylobacter species, in particular Campylobacter jejuni, are likely pathogenic in dogs but colonization occurs more commonly. ${ }^{10}$ Limited information is available on the nature and duration of immunity after infection, but long-term infection and re-infection with different strains without symptoms indicates a lack of protective immunity in dogs. ${ }^{11}$

While not a commonly cited phenomenon, genetically proven transmission of Campylobacter jejuni from a puppy to a baby has been documented. ${ }^{12}$ Given a lack of data showing transmission of Campylobacter from animals to vulnerable older population groups, this may be a useful analogy given that extremities of age are recognized periods of increased susceptibility to infection. ${ }^{13}$

\section{(2) Transmissibility of the bacterium}

Modelling in humans suggests a $5-50 \%$ probability of infection after a dose of 100 organisms and a 50$80 \%$ probability of infection with 10000 organisms; $^{14}$ human challenge studies have shown the infectious dose to be as low as 500 organisms. ${ }^{15}$ In humans, excretion 
of bacteria in faeces may occur for several weeks after clinical recovery with long-term carriage observed in immune-deficient patients. ${ }^{16}$

\section{(3) Contact with the puppy}

When an individual dog carries Campylobacter jejuni, the risk of transmission may be high depending on factors such as the level of contact between the dog and people. ${ }^{17}$ Investigators developed an ordinal scale to assess the frequency, intensity and duration of contact between cases and the puppy with the assessment performed by an ACF staff member familiar with the affected staff and residents. The results showed most contact instances were unplanned, involved occasional patting of the puppy and occurred no more than once or twice per week.

\section{(4) Infection control and cleaning processes}

Inspections conducted by public health staff did not identify any specific infection control issues. However, these inspections were carried out before the puppy was identified. No issues were found with access and availability of hand washing facilities, and residents had access to either a personal or a shared bathroom. Additional hand washing and sanitizer stations were located throughout the facility for staff, resident and visitor use. While strict enforcement of hand washing remains the most important hygiene measure following animal contact, there could be no certainty that residents had washed their hands (or used a hand sanitizer) after contact with the dog.

\section{(5) Animal management}

Animal entry to the facility was discretionary, with the facility only requiring an informal evaluation of an animal's medical, social and behavioural suitability. Notably, the facility's animal policy permitted animal entry to food service areas provided that the animal did not interfere with processes (e.g. begging for food).

\section{Consequence of infection}

Although generally self-limiting, adverse outcomes in the elderly as a result of Campylobacter infection do occur. Following these outbreaks, one resident case died, while another required hospitalization for management of ongoing gastroenteritis. Both had laboratory-confirmed campylobacteriosis. Although deaths in ACFs are not unexpected events, these findings are consistent with research showing the highest mortality for Salmonella and Campylobacter infections in the elderly occurs in the period shortly after illness. ${ }^{18}$ Mortality data also shows the standardized mortality ratio (SMR) for Campylobacter cases $\geq 65$ years of age to be $200 \%$ higher than the general population at one month post-infection (SMR 3.0, $95 \% \mathrm{Cl}: 2.0-4.3) .{ }^{19}$

Based on the overall risk assessment and using a likelihood consequence matrix (Figure 2), ${ }^{20}$ the panel determined that the puppy posed a high risk to residents' health.

\section{Risk management}

The panel also considered several other activities that could be undertaken to assist with reducing risk to an acceptable level.

\section{(1) Eliminating infection in the puppy using antibiotics}

A review of bacterial enteritis in dogs and cats ${ }^{10}$ identified that veterinary guidelines were lacking on the efficacy of antimicrobial therapy and suggested human guidelines be considered with treatment reserved for moderate to severe cases and early infections. Erythromycin, fluoroquinolones and second generation cephalosporins have been used for treatment of diarrhoeic dogs, but the efficacy is unclear. ${ }^{10}$ Treatment of carriers has been considered in high-risk environments, such as pet stores or kennels, but the risk of re-exposure limits the chance of efficacy and increases the risk of antibiotic resistance. ${ }^{10}$ Animals treated with antibiotics could also potentially serve as reservoirs for antibiotic-resistant microorganisms introduced to the facility while the animal is present. ${ }^{5}$

\section{(2) Infection control and animal management considerations}

International guidelines ${ }^{21}$ recommend that only adult animals that are a part of a formal AAl programme should be permitted into a setting such as an ACF; dogs in particular need to be at least one year of age but ideally two years of age or older. An animal that is part of an AAI programme should be more temperamentally suited to the environment, providing greater assurance of behavioural 
control. Criteria for assessing temperamental suitability might include how the animal reacts to strangers, loud or novel stimuli, threatening voices or gestures, crowding, excessive patting or restraint, the presence of other animals and the handler's commands. ${ }^{21}$

The presence of a handler or other supervision while a dog is interacting with residents is an important consideration. From an infection control perspective this can assist with restricting animal movements into sensitive areas such as kitchens, dining rooms, laundries, sterile supply and medication preparation areas. ${ }^{5}$ The handler or supervisor can also take responsibility for ensuring that residents sanitize or wash their hands both before and after contact with the animal. If there is a toileting incident involving the animal, avoidance of direct contact with animal faeces should be stressed; the use of gloves and leak-resistant bags to discard absorbent material used in the cleaning process is recommended. ${ }^{5}$

\section{Recommendations}

After considering the panel's findings of a high risk to elderly residents and the options available for the management of that risk, public health authorities adopted the following recommendations as proposed by the panel:

1. that the puppy must not return to the facility until it is at least one year of age;

2. that the puppy must have its behaviour and temperament assessed as being appropriate for an aged-care environment; and

3. that the facility must revise its infection control and animal policies to ensure zoonotic disease risks are considered.

\section{DISCUSSION}

An aged-care outbreak of campylobacteriosis is an anomaly requiring thorough investigation to identify and eliminate ongoing risk. Under such circumstances investigators need to be cognizant of novel causes of transmission. While zoonotic transmission is less commonly reported, puppies have been identified as a recognized risk factor for campylobacteriosis, ${ }^{3,7}$ and there is extensive evidence detailing shedding by asymptomatic dogs, particularly younger animals. ${ }^{8,9}$

In view of this known risk and the potential for adverse health outcomes among elderly persons following infection, ${ }^{18,19}$ a precautionary approach was adopted and the puppy was excluded from the facility pending further investigation and expert consultation. The subsequent isolation of Campylobacter jejuni in the canine stool sample was not entirely unexpected; however, the fact it was a drug-resistant organism from an animal living amidst a vulnerable population was of concern.

The investigation did face challenges, in particular having only a limited number of viable specimens for comparative testing. Although direct zoonotic transmission was not demonstrated, there was no evidence to suggest either person-to-person or foodborne transmission routes were involved. It is plausible that the puppy, as the putative source of infection, may have been colonized with a variety of genetically distinct Campylobacter organisms that were being intermittently shed. ${ }^{8}$ There was also a temporal link between the animal's arrival and removal and the commencement and cessation of cases.

\section{CONCLUSION}

This risk assessment and public health investigation both highlight the need for greater awareness of zoonotic transmission of Campylobacter and the potential for adverse outcomes among a vulnerable population, namely frail elderly persons living in aged-care. ACFs need to adopt and enforce policies that recognize zoonotic risk and restrict inappropriate animal access. Puppies and young dogs should not be considered or permitted as companion animals in ACFs due to their high rates of Campylobacter carriage, their social immaturity, the susceptibility of elderly residents to infection and poor outcomes.

\section{Ethics statement}

Ethics approval to conduct the investigation and risk assessment was not sought as the work was being conducted as part of a public health response. 


\section{Conflicts of interest}

None declared.

$$
\text { Funding }
$$

OzFoodNet is funded by the Australian Government Department of Health.

\section{Acknowledgements}

The authors wish to acknowledge the following groups for their assistance with the investigation: staff at the Communicable Disease Control section at the ACT Health Protection Service; Environmental Health officers at the ACT Health Protection Service; staff at the ACT Government Analytical Laboratory; staff at ACT Pathology; staff at Capital Pathology; staff at the Microbiological Diagnostic Unit, University of Melbourne; and residents and staff at the affected aged-care facility.

\section{References:}

1. OzFoodnet Working Group. Monitoring the incidence and causes of diseases potentially transmitted by food in Australia: Annual Report of the OzFoodNet network, 2009. Communicable Diseases Intelligence Quarterly Report, 2010, 34:396-426. pmid:21413526

2. Stafford RJ et al.; OzFoodNet Working Group. A multi-centre prospective case-control study of campylobacter infection in persons aged 5 years and older in Australia. Epidemiology and Infection, 2007, 135:978-988. doi:10.1017/S0950268806007576 pmid: 17134530

3. Stafford RJ et al.; OzFoodNet Working Group. Populationattributable risk estimates for risk factors associated with Campylobacter infection, Australia. Emerging Infectious Diseases, 2008, 14:895-901. doi:10.3201/eid1406.071008 pmid:18507899

4. Kirkpatrick BD, Tribble DR. Update on human Campylobacter jejuni infections. Current Opinion in Gastroenterology, 2011, 27:1-7. doi:10.1097/MOG.0b013e3283413763 pmid:21124212

5. Sehulster LM et al. Guidelines for environmental infection control in health-care facilities. Recommendations from $\mathrm{CDC}$ and the Healthcare Infection Control Practices Advisory Committee (HICPAC). Chicago, American Society for Healthcare Engineering/ American Hospital Association, 2004.

6. DiSalvo $\mathrm{H}$ et al. Who let the dogs out? Infection control did: utility of dogs in health care settings and infection control aspects. American Journal of Infection Control, 2006, 34:301-307. doi:10.1016/j.ajic.2005.06.005 pmid:16765210
7. Tenkate TD, Stafford RJ. Risk factors for campylobacter infection in infants and young children: a matched case-control study. Epidemiology and Infection, 2001, 127:399-404. doi:10.1017/ S0950268801006306 pmid:11811871

8. Hald B et al. Longitudinal study of the excretion patterns of thermophilic Campylobacter spp. in young pet dogs in Denmark. Journal of Clinical Microbiology, 2004, 42:2003-2012. doi:10.1128/JCM.42.5.2003-2012.2004 pmid:15131162

9. Torre E, Tello M. Factors influencing fecal shedding of Campylobacter jejuni in dogs without diarrhea. American Journal of Veterinary Research, 1993, 54:260-262. pmid:8430936

10. Weese JS. Bacterial enteritis in dogs and cats: diagnosis, therapy, and zoonotic potential. The Veterinary Clinics of North America. Small Animal Practice, 2011, 41:287-309. doi:10.1016/j. cvsm.2010.12.005 pmid:21486637

11. Koene MGJ et al. Strain variation within Campylobacter species in fecal samples from dogs and cats. Veterinary Microbiology, 2009, 133:199-205. doi:10.1016/j.vetmic.2008.06.022 pmid: 18678447

12. Wolfs TFW et al. Neonatal sepsis by Campylobacter jejuni: genetically proven transmission from a household puppy. Clinical Infectious Diseases, 2001, 32:E97-99. doi:10.1086/319224 pmid:11229867

13. Crossley KB, Peterson PK. Infections in the Elderly. In: Principles and Practice of Infectious Diseases, Mandell GL, Bennett JE, and Dolin R, Editors. Philadelpia, Churchill Livingstone Elsevier, 2010, 3857-3864.

14. Heymann DL, editor. Control of Communicables Manual 19th edition. Washington, DC, American Public Health Association, 2008, 94-98.

15. Kothary MH, Babu US. Infective Dose of Foodborne Pathogens in Volunteers: A Review. Journal of Food Safety, 2001, 21:49-68. doi:10.1111/j.1745-4565.2001.tb00307.x

16. Blaser MJ, Engberg J. Clinical aspects of Campylobacter jejuni and Campylobacter coli infections. In: Campylobacter, Nachamkin I, Szymanski CM, and Blaser MJ, Editors. Washington, DC, American Society for Microbiology, 2008, 99-121.

17. Parsons BN et al. Typing of Campylobacter jejuni isolates from dogs by use of multilocus sequence typing and pulsed-field gel electrophoresis. Journal of Clinical Microbiology, 2009, 47:34663471. doi:10.1128/JCM.01046-09 pmid:19794053

18. Gradel KO et al. Morbidity and mortality of elderly patients with zoonotic Salmonella and Campylobacter: a populationbased study. The Journal of Infection, 2008, 57:214-222. doi:10.1016/j.jinf.2008.06.013 pmid:18656265

19. Ternhag $A$ et al. Mortality following Campylobacter infection: a registry-based linkage study. BMC Infectious Diseases, 2005, 5:70. doi:10.1186/1471-2334-5-70 pmid:16162289

20. ACT Government Health Directorate. Risk Management Guidelines, Internal Audit and Risk Management. Canberra, Department of Health, 2012.

21. Lefebvre SL et al.; Writing Panel of Working Group. Guidelines for animal-assisted interventions in health care facilities. American Journal of Infection Control, 2008, 36:78-85. doi:10.1016/j. ajic.2007.09.005 pmid:18313508 